\title{
Los sistemas integrados de producción agropecuaria como alternativa agroecológica (experiencia Cubana)
}

Integrated systems of agricultural production and agroecological alternative (Cuban experience)

\author{
Iglesias JM, Funes F, Machado $\mathrm{H}$ y Soca $\mathrm{M}^{1}$ \\ ${ }^{1}$ Estación Experimental de Pastos y Forrajes "Indio Hatuey", Matanzas, Cuba \\ iglesias@indio.atenas.inf.cu
}

CONFERENCIA DEL I SEMINARIO INTERNACIONAL DE GANADERÍA AGROECOLÓGICA REALIZADO EN VILLAVICENCIO COLOMBIA, 11 y 12 de noviembre de 2010

\section{RESUMEN}

Los sistemas especializados de producción requieren generalmente de un flujo exógeno de recursos y su mantenimiento y estabilidad no dependen de su capacidad propia de sustento. En cambio, los sistemas productivos agroecológicos, que miran hacia los procesos, muestran mayor capacidad de sostenimiento, generando sinergias que permiten potenciar las capacidades naturales de producir alimentos de origen vegetal y animal y de restaurar el equilibrio productivo, ecológico y económico, ante la influencia negativa de cualquier factor interno o externo. Un sistema no es considerado la suma de sus partes, sino el resultado del todo, formado por las partes. En los sistemas agroecológicos se implanta no solo la racionalidad biológica del funcionamiento del suelo, las plantas y los animales, sino que se relaciona con el hombre y su entorno social, económico y político. Aquí se desecha la visión antropocéntrica del mundo y toma lugar la visión biocéntrica, que explica el funcionamiento y evolución de cualquier cambio. Otro aspecto a tener en cuenta son las técnicas tradicionales y los conocimientos locales. Las experiencias acumuladas, los conocimientos tradicionales y locales, incluso los empíricos, en la actividad agropecuaria se trasmiten de generación en generación y son de mucha importancia para lograr resultados productivos y eficientes.

Palabras clave: Sistemas agropecuarios, ecología, Cuba. 


\section{ABSTRAC}

Specialized production systems generally require exogenous flow of resources and their maintenance and stability does not depend on its ability to sustain itself. In contrast, agroecological production systems, facing processes, show greater carrying capacity, generating synergies that may enhance natural abilities to produce food of plant and animal origin and to restore balance productive, ecologically and economically, to the influence refusal of any internal or external factor. A system is considered the sum of its parts, but the result of all, made by the parties. In agroecological systems implanted rationality not only biological functioning of soil, plants and animals, but is related to man and their social, economic and political. Here is discarded anthropocentric view of the world and takes place biocentric vision, which explains the functioning and evolution of any changes. Another aspect to consider are the traditional techniques and local knowledge. The experience acquired traditional and local knowledge, including empirical, agricultural activity is transmitted from generation to generation and are very important to achieve productive and efficient results.

Keywords: Agricultural systems, ecology, Cuba.

\section{INTRODUCCIÓN}

En la orientación biológica dinámica de la agroecología, los ritmos biológicos, la periodicidad entre día y noche, las fases lunares y constelaciones astrales, se aprovechan conscientemente en la producción agrícola y ganadera, y sus efectos se manifiestan evidentemente, en la calidad de los productos y en el resultado económico. En la agricultura tradicional muchas veces nos enfrentamos con el balance energético negativo, donde se invierte más energía que el resultado final del proceso de producción. Esta energía es de fuentes no renovables como petróleo, gas natural o carbón; mientras que en la agricultura ecológica, sostenible domina el principio del uso mínimo de energía externa y del balance energético positivo. Este se logra por el ahorro de energía en la labranza, así como por el uso de energía solar en el calentamiento del agua, la calefacción y la deshidratación 
de los productos, la generación de electricidad por medio de celdas fotovoltaicas, el bombeo de agua por medio de energía eólica y el uso de biocombustibles.

Los principios agroecológicos que permiten la sustentabilidad biológica y la viabilidad económica de las fincas agropecuarias son: Diversificación espacial y temporal, integración de la producción animal y vegetal, mantenimiento de altas tasas de reciclaje de los desechos animales y vegetales y optimización del uso del espacio (Figura 1).

\section{PRODUCTOS AGROPECUARIOS}

En el caso de Cuba se han experimentado y llevado a cabo una serie de combinaciones y modelos de agricultura ecológica, principalmente en los predios de los agricultores privados o asociados a cooperativas de producción agropecuaria, los cuales han tenido éxito en el tiempo y sus resultados se han venido diseminando a través de los programas de transferencia de tecnologías "De campesino a campesino", "Fitomejoramiento participativo", "Iniciativa para el desarrollo local", "Programa de Agricultura Urbana", "Movimiento Agroecológico de la ANAP", "Popularización del arroz y otros (Funes et al., 2002) y con la ayuda de asociaciones y organismos como la Sociedad de Agricultura Orgánica, la Asociación Cubana de Producción Animal", la Asociación Cubana de Trabajadores Agrícolas y Forestales" (Tabla 1).

En los últimos años se ha hecho énfasis en la reconversión de los sistemas de producción estatales, los cuales tienen las limitaciones de que siempre dependieron de los insumos externos provenientes del estado y poseen grandes extensiones de tierra, con una agricultura o ganadería de monocultivo principalmente, lo que ha provocado una explotación indiscriminada de los recursos naturales (bosques, suelos) y causado enormes impactos ambientales sobre el suelo y la pérdida de biodiversidad (Treto et al., 2002; Funes y Monzote, 2004).

En este proceso de reconversión, denominado "modelo de sustitución de insumos", se visualiza una disminución de las áreas agrícolas dedicadas a la 
producción de productos de exportación, cambios profundos de las estructuras productivas (Tabla 6), una mayor orientación hacia la autosuficiencia alimentaria y una mayor preocupación por la protección del medio ambiente (Funes et al., 2002).

Tabla 1. Tenencia (\%) de la tierra en Cuba (1989-2006)

\begin{tabular}{cccc}
\hline Sector & $\mathbf{1 9 8 9 - 1 9 9 2}$ & $\mathbf{1 9 9 3}$ & $\mathbf{2 0 0 6}$ \\
\hline Estado & & 47.0 & \\
Otras organizaciones & 83.0 & 9.0 & 33.1 \\
estatales & & 26.5 & 40.6 \\
UBPC & - & 7.5 & 26.3 \\
CPA & 12 & 10.0 & 2 \\
Privados & &
\end{tabular}

No obstante, aún más del $55 \%$ de los alimentos que consume la población cubana son importados (García, 2003), lo que implica una erogación de alrededor de 1033 millones de USD anuales en la compra de los mismos. Por otra parte, persiste la dependencia en combustibles fósiles y se incrementó en los últimos años el éxodo de las poblaciones rurales hacia las ciudades (solo el 25\% permanece en áreas rurales, de ellos el $11 \%$ vinculado al sector agropecuario).

Esto nos indica que el modelo de sustitución de insumos tampoco es la vía adecuada para una producción agrícola sostenible, ya que no es lo suficientemente versátil, descentralizada y factible para adaptarse a las disímiles condiciones de suelo, clima, características agroecológicas, gustos y percepciones de cada productor (Funes y Monzote, 2006), por lo que se hace necesaria una definitiva transición agroecológica, basándose en los resultados exitosos de los productores que asumieron ese modelo como pauta en su desarrollo agropecuario. Los casos más exitosos de integración ganadería-agricultura se presentan en las Figura 1 y 2, Fotografías 1 a 11.

Cualquiera de los avances mencionados anteriormente ha significado una serie de trabajos de investigación y experimentación con productores en los que no siempre se pueden atender todos los requisitos del método científico clásico y 
donde la experiencia acumulada del campesino juega un papel determinante en la toma de decisiones y en el cambio de los diseños agropecuarios.

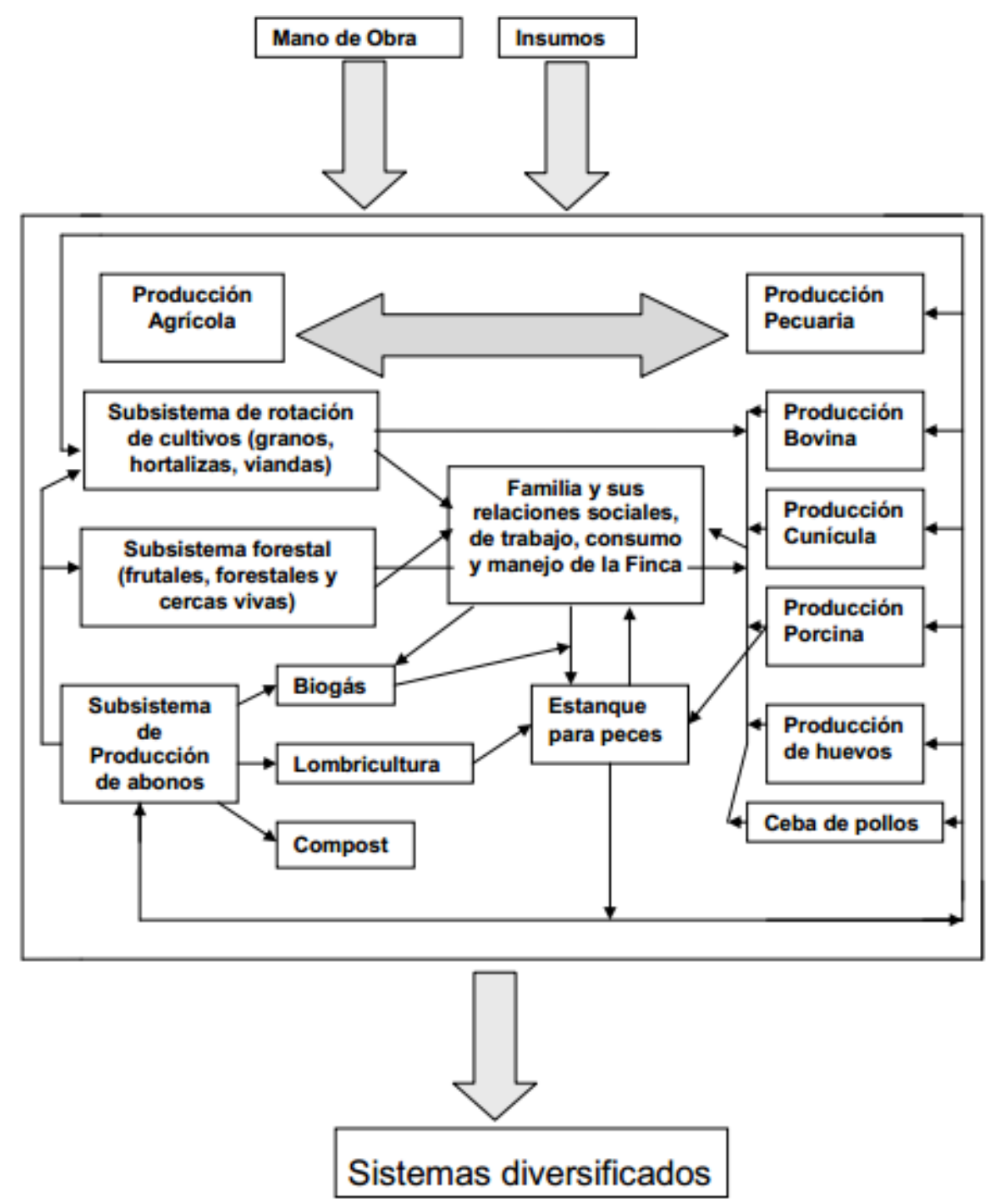

Figura 1. Interacciones entre los diferentes subsistemas de una finca familiar integrada

A pesar de los esfuerzos queda mucho por hacer, tanto desde el punto de vista productivo, como de lo relacionado con el extensionismo y la superacióncapacitación de los campesinos, empresarios, promotores y facilitadores del cambio hacia una agricultura racional, biodiversa y de bajos insumos. También se debe reconocer que, en la mente de los decisores de políticas agrarias y de muchos productores aún prima, en sentido general, el enfoque agronómico sobre 
el ecológico en el manejo y la conservación de los suelos, el aumento de la productividad mediante la entrega de nuevas tierras de cultivo y el desmonte de áreas vírgenes, la utilización de fertilizantes para compensar la pérdida de la fertilidad, la introducción de variedades mejoradas y el uso de maquinarias sofisticadas para hacer más eficientes las tareas agrícolas.

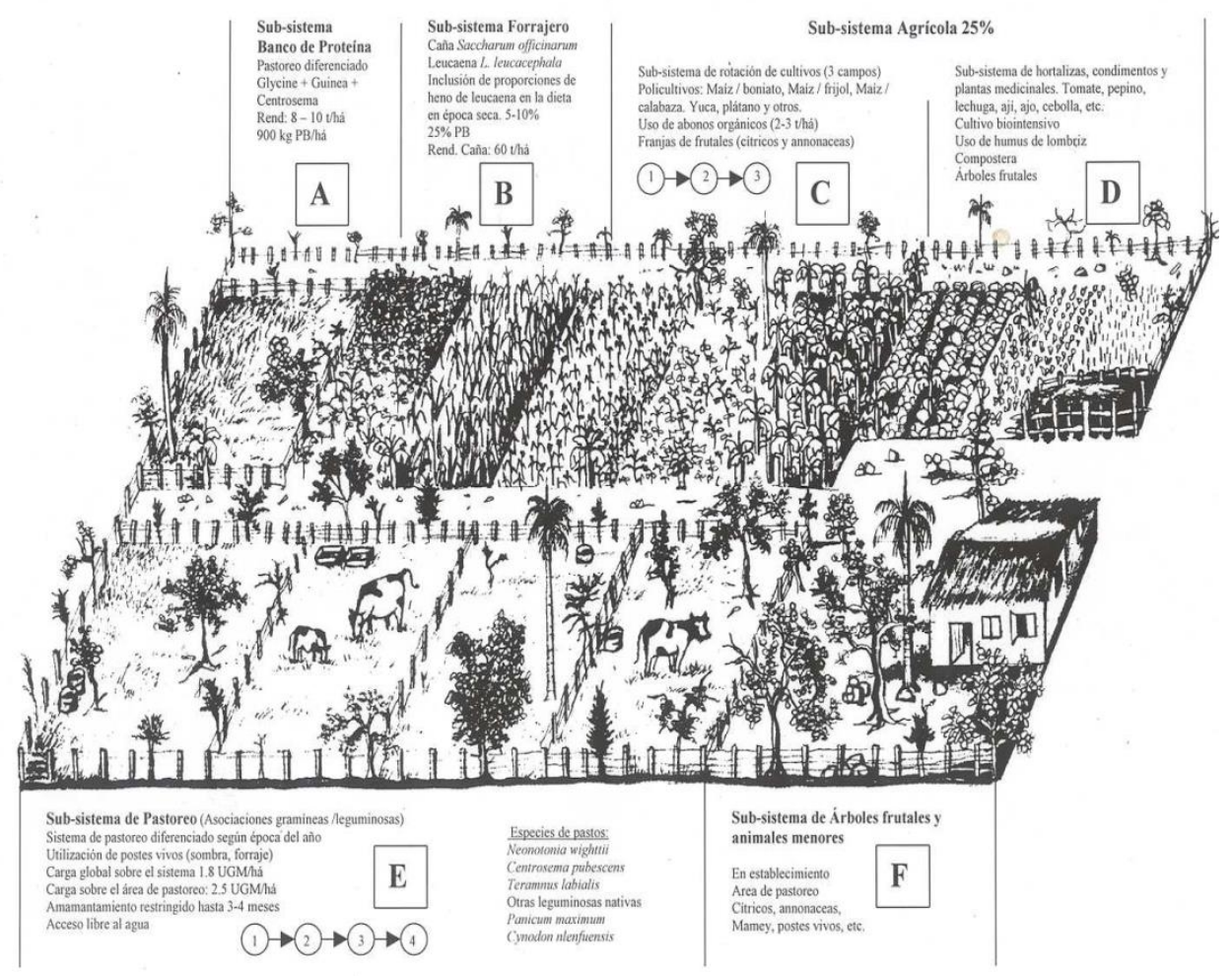

Fuente: Funes y Monzote, (2006).

Figura 2. Sistemas diversificados con utilización de la ganadería en el $75 \%$ del área

Esta orientación tecnológica es insostenible económicamente en las condiciones actuales e insustentable desde el punto de vista ecológico, en tanto compromete las perspectivas futuras de desarrollo. El modelo agrícola moderno (convencional) ha subestimado la complejidad de los procesos ecológicos y sobrestimado la sustitución de estos procesos por la tecnología, generando desafíos ambientales que manifiestan la fragilidad del desarrollo alcanzado. Realmente, hoy se apela a la agroecología porque se necesita como nunca antes lograr reducir los costos de producción, una mayor diversificación de las posibilidades reales con que cuentan los sectores agroproductivos, potenciando el uso óptimo de los recursos laborales. 
Se hace necesario trabajar para que esto no sea una táctica coyuntural dada la escasez de insumos, sino que se entienda la pertinencia de esta forma de hacer agricultura como estrategia a largo plazo dentro del sistema agrícola.

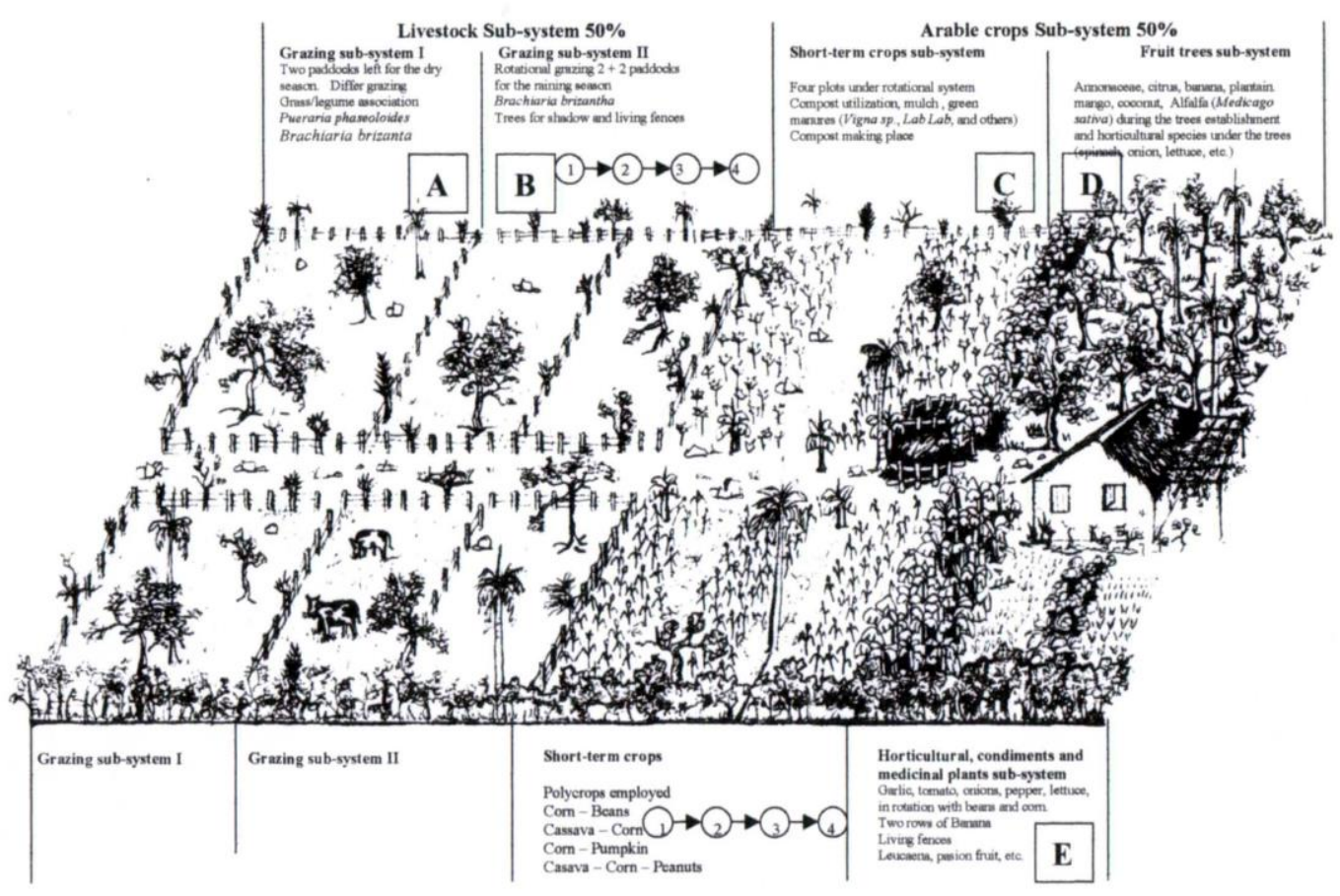

Fuente: Funes y Monzote, (2006)

Figura 3. Sistemas diversificados con utilización de la ganadería en el 50\% del área

Ante el cambio en las condiciones de la agricultura cubana es necesario crear sistemas agrícolas de bajo uso de insumos externos, de bajo costo, de alto uso de recursos locales diversificados y eficientes en el uso de la energía, capaces de presentar rendimientos sostenidos en el tiempo, mediante tecnologías económicamente balanceadas; es decir, que busquen un manejo eficiente del conjunto del sistema agroecológico; pero los cambios deben ser graduales, tomando conjuntamente las medidas para restablecer la fertilidad de los suelos y el equilibrio biológico, a la vez que disminuye la dependencia de los fertilizantes químicos. Las transformaciones rurales que tienen lugar en la actualidad, apuntan hacia la readaptación del actual modelo convencional, sobre la base de la sustitución de insumos y de un mayor protagonismo de los sectores 
agroproductivos para elevar los rendimientos productivos y la eficiencia económica.

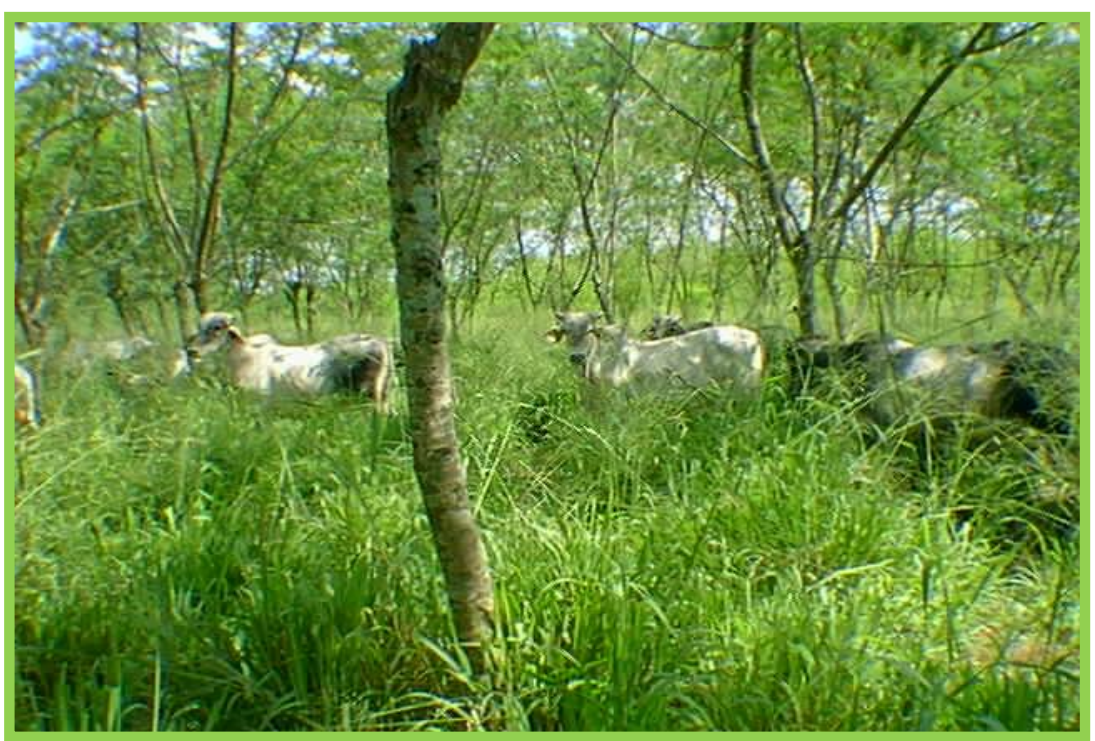

Fuente: Pérez, (2007); Iglesias (2003).

Fotografía 1. Sistemas pastoriles y silvopastoriles integrados a la producción de semillas de pastos y arbóreas
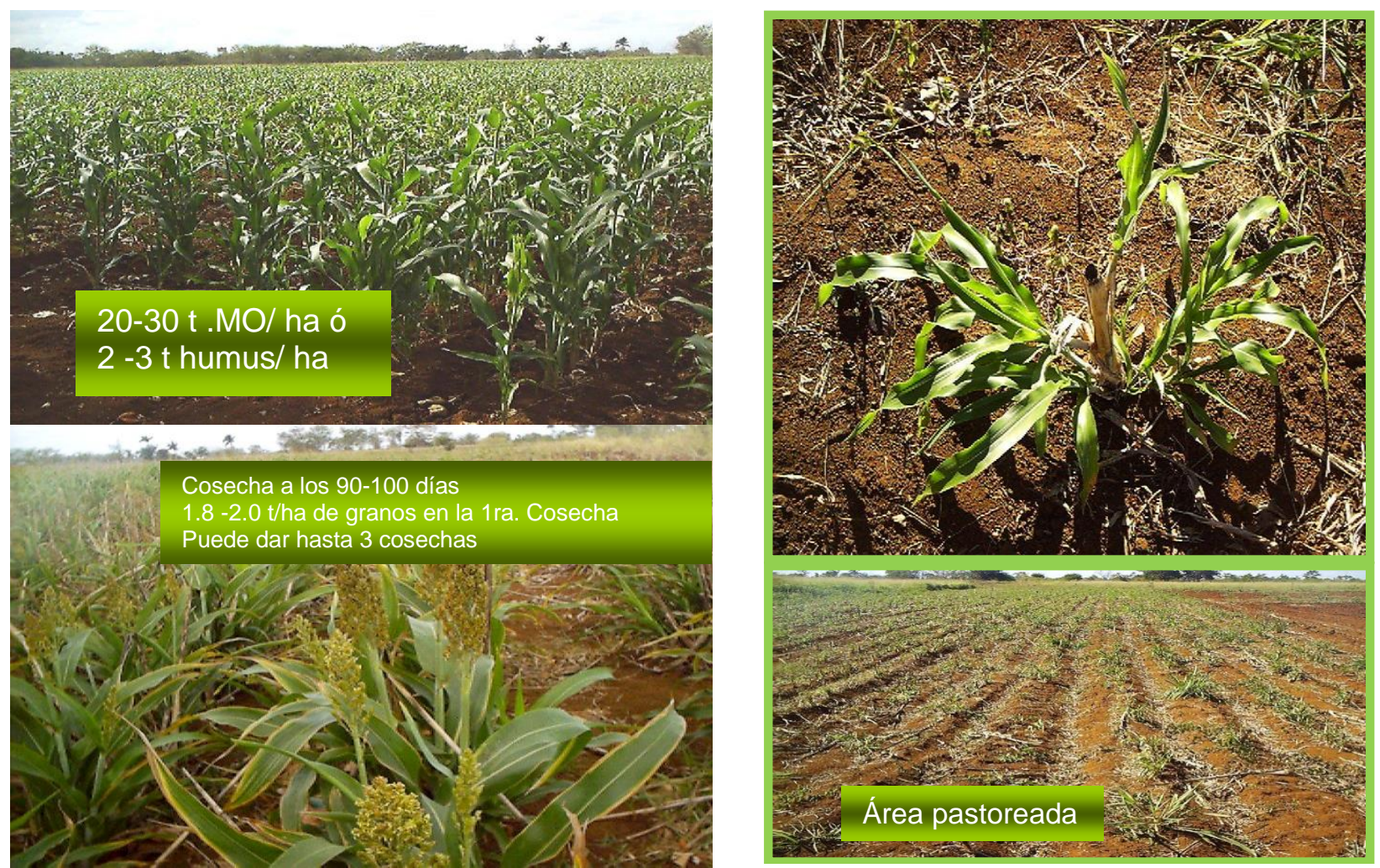

Fuente: Pérez, (2007); Iglesias (2003).

Fotografía 2. Sistemas pastoriles y agrosilvopastoriles con producción de cultivos anuales en áreas segregadas, así como granos de interés forrajero 

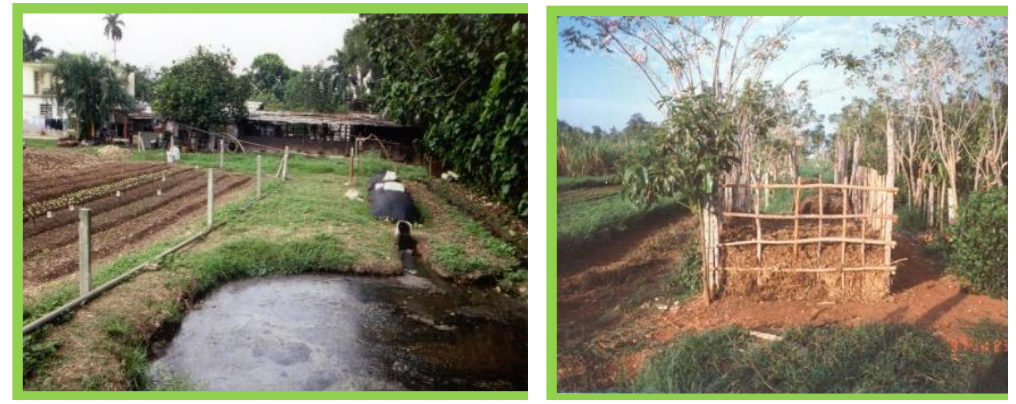

Fuente: García, (2004).

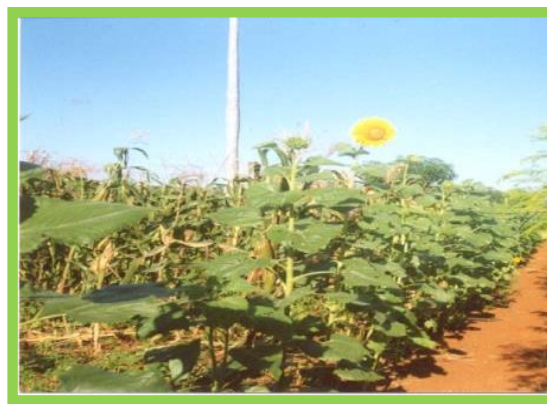

Fotografía 3. Biotransformación de los residuales porcinos a través de la producción de compost y la vermicultura y su uso en la producción de granos forrajeros

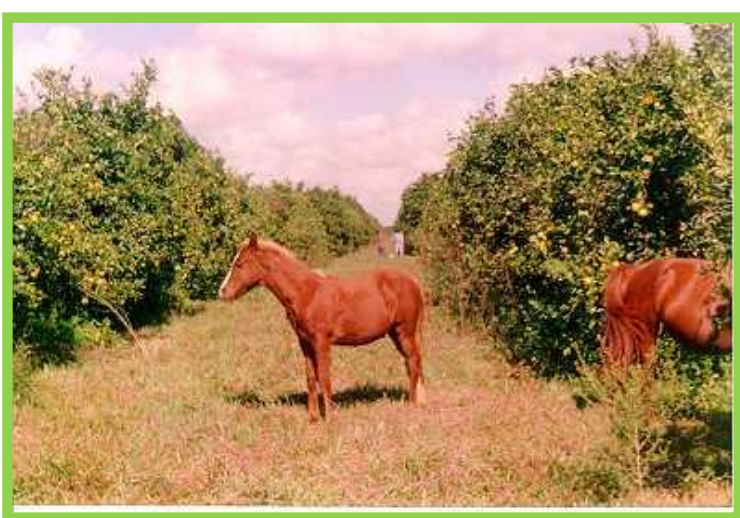

Fuente: Simón y Francisco, (2000).

Fotografía 4. Cultivos de cobertura en áreas de cocales y cítricos, combinado con ganadería ovino-caprina y equina

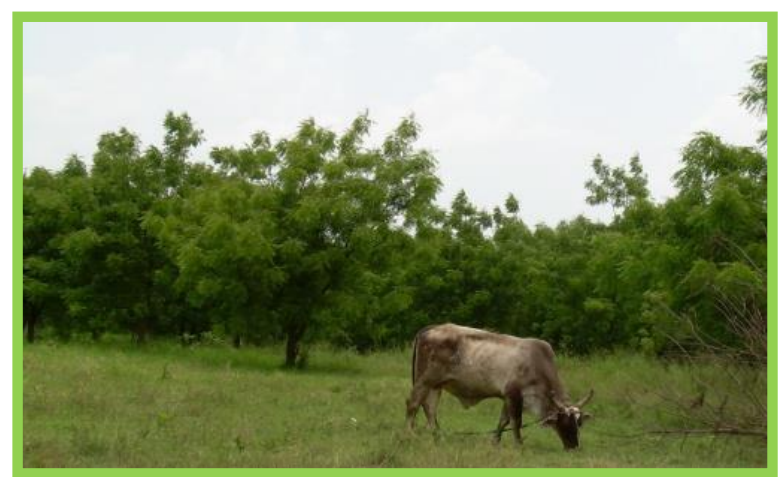

Foto 6. Uso de bioderivados del árbol del Nim para el control de parásitos que afectan los animales de cría

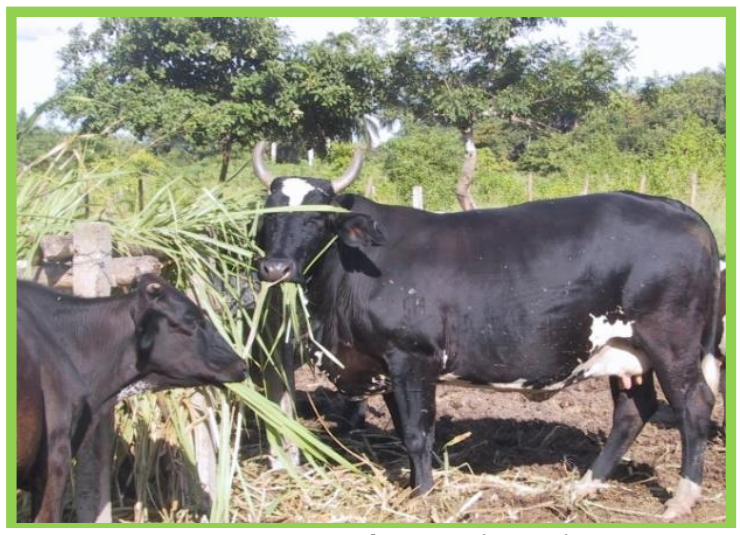

Fuente: Hernández, (2005).

Fotografía 5. Producción de caña forrajera con el uso de arbóreas como abono verde

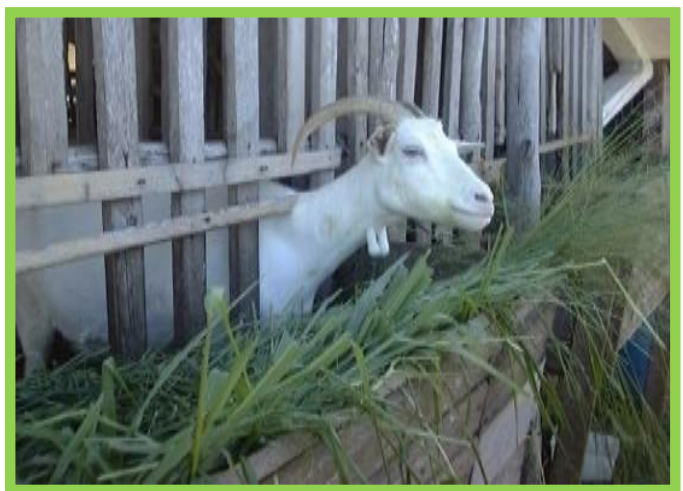

Fuente: Suárez, (2004).

Fotografía 7. Producción agroecológica de leche de cabras en estabulación a partir de forrajes y residuos de las cosechas de una finca diversificada 


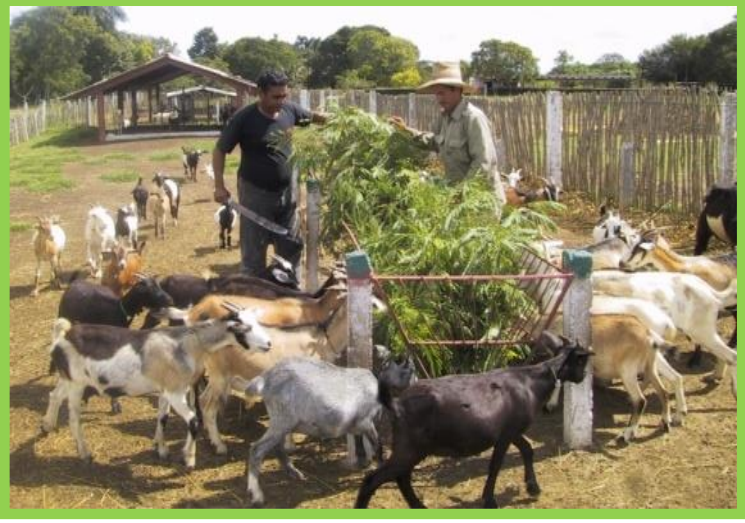

Fotografía 8. Producción agroecológica de leche de cabras en pastoreo, forraje de arbóreas y residuos de cosechas diversas

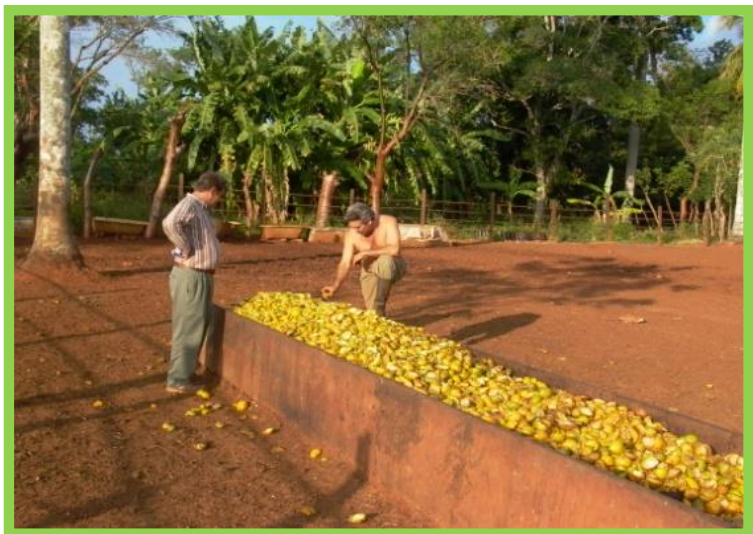

Fuente: Ojeda, (2006).

Fotografía 9. Producción animal a partir de residuales frescos y preelaborados (ensilaje, harinas, pellets) de la industria citrícola
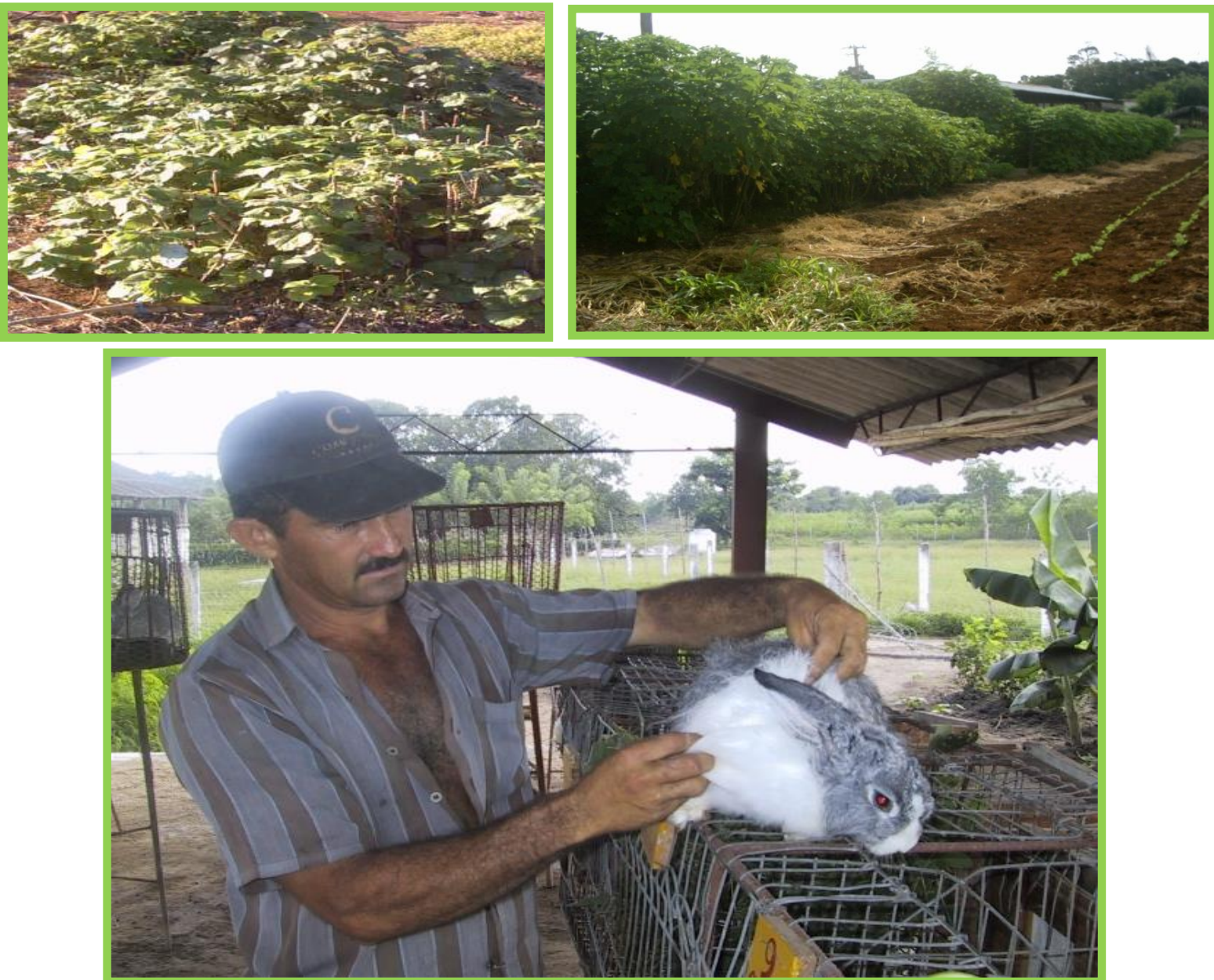

Fuente: Pérez, (2007); Iglesias, (2006)

Fotografía 10. Producción cunícola y porcina con piensos locales y forrajeras proteicas 

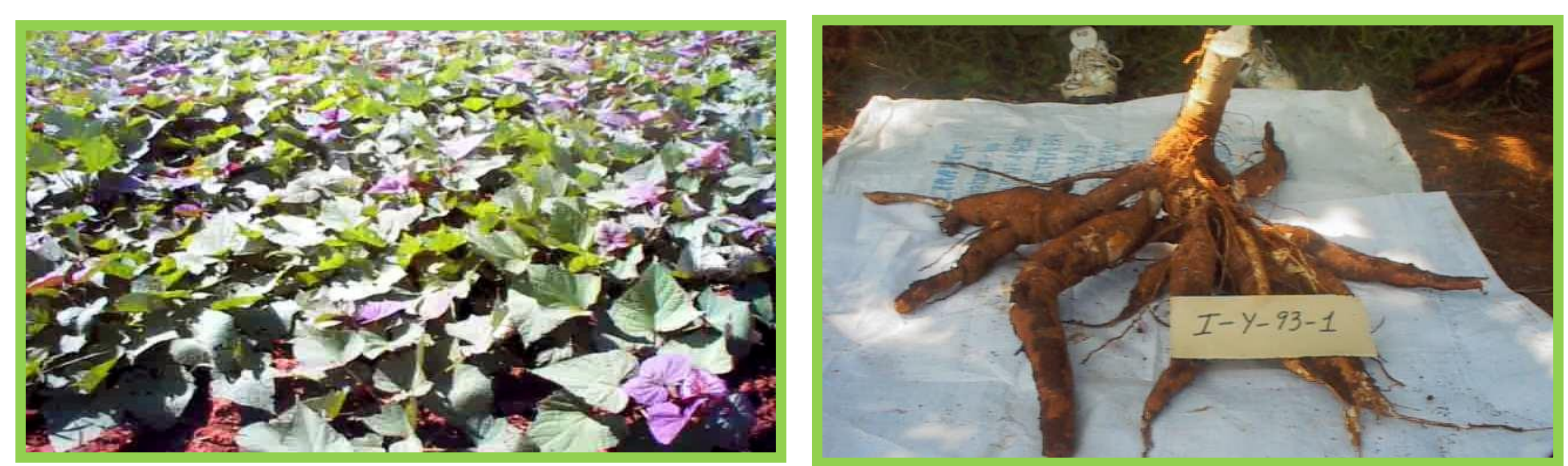

Fuente: García y Medina, (2005).

Fotografía 11. Uso de raíces y tubérculos en la alimentación animal

\section{CONSIDERACIONES FINALES}

- Teniendo en cuenta el limitado avance de las prácticas agroecológicas, se hace necesario de inmediato trazar una estrategia agraria de base agroecológica teniendo en cuenta las condiciones regionales y el potencial agrícola autóctono, aprovechando la generación propia de tecnologías y estableciendo la subordinación municipal de la agricultura.

- Se debe fomentar la reconversión tecnológica tratando de sustituir gradualmente las tecnologías importadas por tecnologías locales más sustentables económicamente priorizando la biotecnología artesanal para la producción de biofertilizantes y biopesticidas. Conjuntamente se debe recalificar la fuerza de trabajo con base en la utilización de tecnologías blandas.

- Es necesario el paso gradual de las entregas de insumos agropecuarios de formas centralizadas a las descentralizadas con participación de los mecanismos de mercado.

- De no materializarse estas y otras propuestas de carácter político-organizativo no tocadas en este artículo será imposible modificar sustancialmente los resultados tradicionales del sector, al no lograrse estimular el uso intensivo de la tierra como condición básica para el logro de una agricultura sostenible. 


\section{REFERENCIAS BIBLIOGRÁFICAS}

1. Funes $M H$, García $L$, Bourque $M$, Perez $N$, Rosset $P$. Sustainable agricultura a resistance. Transforming food production in Cuba. Food first. ACTAF, CESA USA, 2002.

2. Funes M F. Towards sustainable agriculture in Cuba [Consultado el 28 de agosto 2011]. 2004: 1-24 Disponible http://campus.usal.es/ ehe/Papers/Microsoft\%20Word\%20\%20Towards\%20sustaina ble\%20agriculture\%20in\%20Cuba\%201st\%20August\%5B1\%5D.pdf

3. Funes M H. ¿Sustitución de insumos o agricultura ecológica? LEISA Revista de Agroecología, 2006: 22 (2): 1-4.

4. Funes F, M M. Sistemas agroecológicos y su papel en los países del tecer mundo. Rev. IAA. 2006:10 (3):5-27.

5. García D E. Efecto de los principales factores que influyen en la composición fitoquímica de Morus alba (Linn.). Tesis presentada en opción al título de Master en Pastos y Forrajes. EEPF "Indio Hatuey". Matanzas, Cuba. 120 p. 2003

6. García D. Los metabolitos secundarios de especies vegetales. Pastos y forrajes. 2004:27:1-12.

7. García D, Medina M, Contenido antinutricional de la biomasa comestible en especies forrajeras del género Albizia Zootecnia Trop. 2005.23:(4)222.

8. González L. La extensión agraria en Cuba, algunas reflexiones necesarias. Pastos y forrajes. 2004:27(3):211-218.

9. Iglesias J M. Cría de hembras bovinos en desarrollo en condiciones de silvopastoreo. Pastos y forrajes. 2003:26(1):35-46.

10. Hernández M. Rendimiento forrajero de la caña de azúcar asociadas a leguminosas arbóreas II. Biomasa comestible. Pastos y forrajes 2005:29 (2): 149-153.

11. Iglesias J M. Cría de hembras bovinos en desarrollo en condiciones de silvopastoreo. Pastos y forrajes. 2003:26(1):35-46.

12. Iglesias J M. Sistemas agroforestales en Cuba, algunos aspectos de producción animal. Pastos y forrajes. 2006:29(3):217-235.

13. Kevin B. Wright Advantages and Disadvantages of Online Survey Research. Services Researching Internet-Based Populations Department of Communication.

14. Ojeda F. Estudio de la calidad fermentativa de la morera y la hierba de guinea ensilados en diferentes proporciones. Pastos y forrajes. 2006:29(2):195-202

15. Pérez A. Producción comercial de semilla de Rodhes callede con fertilizantes orgánicos y minerales. Pastos y forrajes. 2001:24(4):291-295.

16. Pérez A. Generación difusión y adopción de tecnologías para la producción beneficio y conservación de semillas de plantas. Forrajeras con enfoque empresarial. Pastos y forrajes. 2007:30(4):399-429.

17. Simón, L, Francisco A. Potencialidades productivas del silvopastoreo. Memorias IV Taller Internacional Silvopastoril "Los árboles y arbustos en la ganadería tropical". EEPF "Indio Hatuey". Matanzas, CubaTomo II. 2000: 467.

18. Suárez J. la gestión de tecnología y la innovación en empresas ganaderas cubanas, para la implementación de la función inventariar. Pastos y forrajes. 2004:27(1):73-90.

19. Treto, E, García, M., Martínez Viera, R., Febles, J. M. Advances in organic sol management In: F. Funes, L. García, M. Bourque, N. Pérez, and P. Rosset, (eds) Sustainable Agriculture and Resistance. Food First, Oakland, CA. 2002:164-169. 